\title{
A Multiwavelength Analysis of the Long-duration Flare Observed on 15 April 2002
}

\author{
Anna Kepa ${ }^{1}$ (D) Barbara Sylwester $^{1}$ (D) \\ Janusz Sylwester $^{1}$ (D) Tomasz Mrozek $^{1,2}$ (D) \\ Marek Siarkowski ${ }^{1}$ (D)
}

Received: 24 May 2019 / Accepted: 7 January 2020 / Published online: 6 February 2020

(C) The Author(s) 2020

\begin{abstract}
We present a multiwavelength analysis of the long-duration flare observed on 15 April 2002 (soft X-ray peak time at 03:55 UT, SOL2002-04-15T03:55). This flare occurred on the disk (S15W01) in NOAA 9906 and was observed by a number of space instruments including the Extreme-Ultraviolet Imaging Telescope on the Solar and Heliospheric Observatory (SOHO/EIT), the RESIK spectrometer onboard the Coronas- $F$ spacecraft, and the Ramaty High Energy Solar Spectroscopic Imager (RHESSI). We have performed a complex analysis of these measurements and studied the morphology and physical parameters characterizing the conditions in flaring plasmas. The $195 \AA$ SOHO/EIT images have been used to study evolution of flaring loops. Analysis of RHESSI data provided the opportunity for a detailed analysis of hard X-ray emission with $1 \mathrm{keV}$ energy resolution. We have used Geostationary Operational Environmental Satellite (GOES) observations for isothermal interpretation of the X-ray measurements. Temperature diagnostics of the flaring plasma have been carried out by means of a differential emission measure (DEM) analysis based on RESIK X-ray spectra. The DEM distributions were calculated based on two methods: Withbroe-Sylwester (WS) and differential evolution (DE). Both of the approaches provided
\end{abstract}

This article belongs to the Topical Collection:

Irradiance Variations of the Sun and Sun-like Stars

Guest Editors: Greg Kopp and Alexander Shapiro

\begin{tabular}{l}
\hline A. Kepa \\
ak@cbk.pan.wroc.pl \\
B. Sylwester \\
bs@cbk.pan.wroc.pl \\
J. Sylwester \\
js@ cbk.pan.wroc.pl \\
T. Mrozek \\
tmrozek@ cbk.pan.wroc.pl \\
M. Siarkowski \\
ms@cbk.pan.wroc.pl \\
Space Research Centre (CBK PAN), Bartycka 18A, Warsaw, Poland \\
Astronomical Institute, University of Wroclaw, Kopernika 11, Wroclaw, Poland
\end{tabular}


similar results. We obtained two-component DEM distributions independent of the evolutionary flare phase. We found that the amount of energy of thermal plasma for this flare is of the order of $10^{30} \mathrm{ergs}$. The values obtained by assuming an isothermal plasma model are lower than those determined from the differential emission measure distributions.

Keywords Corona $\cdot$ Structures $\cdot$ Flares $\cdot$ Spectrum $\cdot$ X-Ray

\section{Introduction}

Long-duration events (LDEs) are a class of flares characterized by slow temporal variations of their X-ray emission (many hours) and decay times significantly longer than the rise phase. LDEs usually occur in long-lived active regions (Kahler, 1977) and their morphological structure is rather complicated. LDEs are often associated with large coronal mass ejections (CMEs) and long-duration post-eruptive energy releases at different wavelengths (Svestka, 1983). They usually have a few hot and bright kernels of emission present along the arcade channel (Jakimiec et al., 1997; Kolomański and Jakimiec, 2002)

The extreme ultraviolet (EUV) emission rises at different phases of the flare evolution (Aschwanden et al., 2009), based on the flare catalogue of the Extreme UltraViolet Imager onboard the Solar Terrestrial Relations Observatory (STEREO/EUVI). Howard et al. (2008) and Kaiser et al. (2008) identified at least three components of EUV emission during the flare. The first one, coincident with enhanced hard X-ray emission, is observed in $79 \%$ of events during the impulsive phase. The second component is related to the cooling of flaring loops, which is accompanied by corresponding changes in the maximum emission from the soft X-rays to EUV temperature range and was noticed during the decay phase of $73 \%$ of flares, when the growth of ultraviolet emission without new flaring X-ray activity was observed. The last effect is the EUV dimming that is caused by the evacuation of expanding plasma during the initial phase of CMEs.

Woods et al. (2011), based on Solar Dynamic Observatory (SDO) observations, reported the fourth flare phase observed during the late phase of flares, which is related to development and brightening of a system of post-eruptive loops of longer length (separated in height from an original flare site).

As concerns the thermal-energy content of the plasma, Jiang et al. (2006) reported for six short flares the thermal energy not exceeding $10^{30}$ ergs. Bạk-Stęślicka, Mrozek, and Kołomański (2011) analyzed physical conditions of flares with rise phase lasting longer than 30 minutes (so-called "slow-long" duration events, SLDEs). The total thermal-energy content for the analyzed flares has been determined there to be $\approx 30^{31} \mathrm{ergs}$ at the maximum.

In this article we present a detailed analysis of a long-duration event on 15 April 2002 when RESIK spectra were available. RESIK was an uncollimated, Polish, bent crystal spectrometer (Sylwester et al., 2005) operating onboard the Russian Coronas-F spacecraft (Kuznetsov, 2014) from 2001 to 2003. The basic components of RESIK were two double spectrometers. Each spectrometer was equipped with two silicon and quartz crystals. The bent crystal causes the radiation beam incident upon the crystal to be reflected at slightly different angles according to Bragg's law, so it was possible to obtain spectra in a selected range of wavelengths instantaneously. The RESIK spectrometer observed simultaneously spectra in four channels that covered the spectral range from $3.3 \AA$ to $6.05 \AA$. The spectra observed in each channel were recorded at 256 spectral bins with data gathering interval (DGI) dynamically determined by the onboard computer according to the level of solar $\mathrm{X}$-ray emission. 
RESIK observations give a possibility of X-ray plasma diagnostics in the temperature range 3-30 MK by determining the differential emission measure distributions (Kepa et al., 2006; Chifor et al., 2007; Sylwester, Sylwester, and Phillips, 2008).

In the determination of differential emission measure distributions we used two algorithms: the Withbroe-Sylwester (WS) approach (Sylwester, Mewe, and Schrijver, 1980) and the differential evolution (DE) method (Storn and Price, 1997). The DEM distributions obtained by the DE method were presented earlier by Kepa et al. (2016). In the present article, we show the results of DE and WS methods as tested on selected synthetic models.

As the flare on 15 April 2002 was also observed by the Extreme Ultraviolet Imaging Telescope (EIT) onboard the Solar and Heliospheric Observatory (SOHO) spacecraft (Delaboudinière et al., 1995) and the Reuven Ramaty High Energy Solar Spectroscopic Imager (RHESSI: Lin et al., 2002), an investigation of flare morphology and the evolution of hard $\mathrm{X}$-ray emissions was possible.

Thanks to observations in different wavelength ranges it was possible to investigate the correlation between the emission from the extreme ultraviolet (EUV) and from soft and hard $\mathrm{X}$-rays. We were also able to compare thermodynamic flare parameters determined assuming the iso-temperature model of plasma with results of multi-temperature analysis. It is known that isothermal models, which allow the determination of temperature and emission values relatively easily, give only average values of these parameters. To examine physical plasma conditions properly, the differential emission measure distributions should be determined.

This article is organized as follows: a description of instruments and observations of the 15 April 2002 flare is presented in Section 2. Results of isothermal and multithermal analysis with application of WS and DE method are shown in Section 3, and the discussion is provided in Section 4.

\section{The 15 April 2002 flare}

We studied the M1.2 GOES class flare (SOL2002-04-15T03:55) that occurred in NOAA Active Region 9906 located at the central part of the solar disk (S15W01). Based on the Solar Geophysical Data report, this event started at 03:05 UT, reached maximum at 03:55 UT, and ended at 05:06 UT. GOES light curves show that emission corresponding to this flare was observed for much longer (see Figure 1). The analyzed flare was preceded by another event at 02:51 UT, looking like a precursor. However, this earlier flare was related to another active region located at the limb (see Figure 2).

Figure 1 displays the temporal profiles of the soft X-rays from GOES $(1-8 \AA)$, RHESSI in the energy range $12-25 \mathrm{keV}$, and the ultraviolet radiation within the area selected (red rectangle in Figure 2) in the $195 \AA$ SOHO/EIT filter. The blue strips on the GOES light curve indicate times when RESIK observations were available. The SOHO/EIT images in $195 \AA$ were taken every 12 minutes only. Respective images have been calibrated using the eit_prep.pro procedure (including corrections for the dark current, exposure time normalization, response correction, etc.). The EIT light curve was derived based on the integration of the signal measured in the area corresponding to the red rectangle marked in Figure 2 after background subtraction (assumed as the level of quiet Sun emission in the immediate vicinity of the considered area/region).

It is seen that the ultraviolet emission appears to have two maxima. The first slight increase of the EUV emission takes place during the rise phase of the soft X-rays and is related to peaking of hard X-rays. The second maximum, much stronger than the first one, was observed during the decay phase of X-ray flare. In this phase the SOHO/EIT light curve is not 


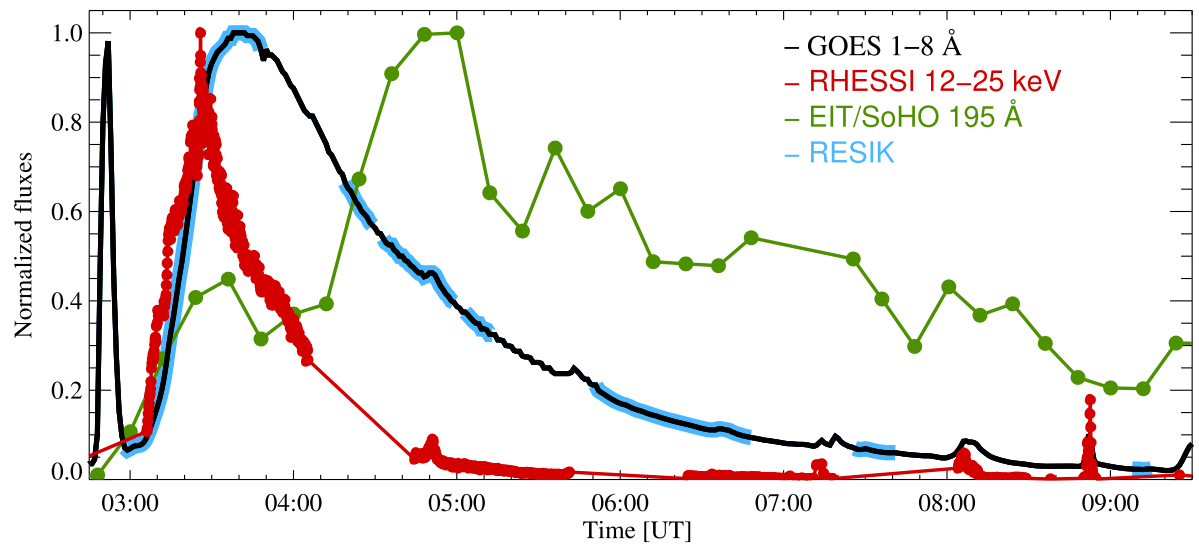

Figure 1 GOES (in black), RHESSI 12-25 keV (red), and SOHO/EIT (green) light curves for the SOL2002-04-15T03:55 flare. The blue strips on the black line (GOES lightcurve) indicate time intervals when RESIK spectra were available.
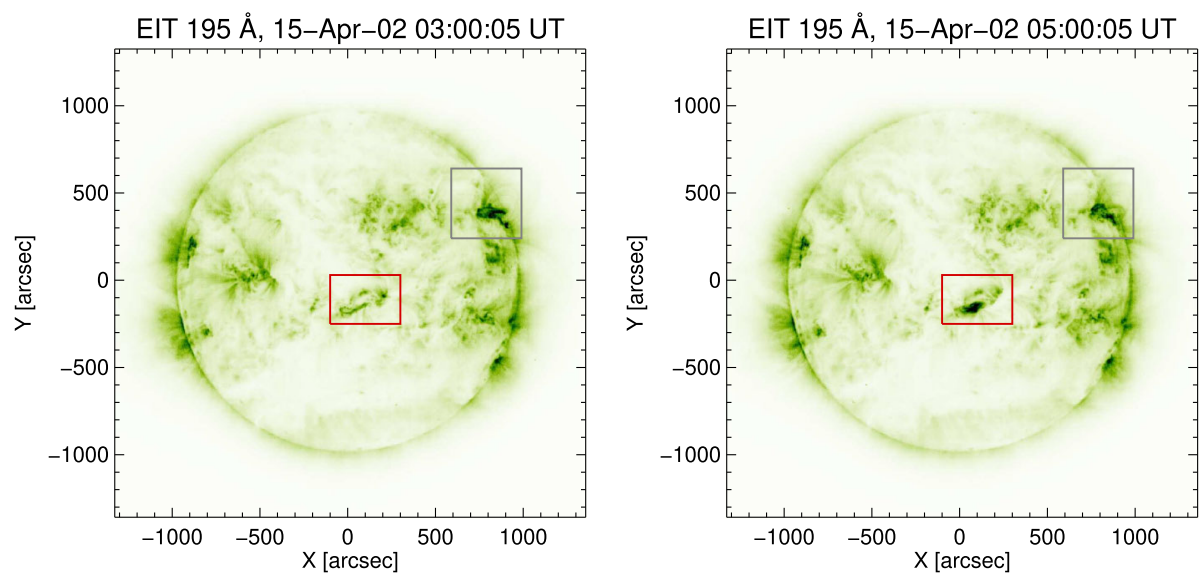

Figure 2 SOHO/EIT images taken in 195 ̊̊ filter at two moments: 03:00:05 UT (left) and 05:00:05 UT (right). The red rectangle indicates the active region in which the analyzed flare occurred. The gray rectangle is related to the location of the preceding event.

monotonic showing a few maxima (the observations were available only every 12 minutes).

A comparison of the light curves in different energy bands allows us determine the time delay between the maxima of the UV $\left(\max _{\mathrm{uv}}\right)$, soft $\left(\max _{\mathrm{sxt}}\right)$, and hard X-ray $\left(\max _{\mathrm{hxt}}\right)$ emissions. For the SOL2002-04-15T03:55 flare the delays are: $\max _{\mathrm{hxt}}-\max _{\mathrm{sxt}} \approx 20$ minutes, $\max _{\mathrm{hxt}}-\max _{\mathrm{uv}} \approx 1$ hour 30 minutes, and $\max _{\mathrm{sxt}}-\max _{\mathrm{uv}} \approx 1$ hour 10 minutes.

RHESSI observed almost the entire main flare phase and the three time intervals during the decay phase (see Figure 1). The emission up to $25 \mathrm{keV}$ was observed.

The RHESSI image reconstruction was made using the PIXON method (Hurford et al., 2002) available in SolarSoft applying grids 3, 4, 5, 6, 8, and 9. The time sequence of the EIT $195 \AA$ Amages with RHESSI contours overplotted obtained in the energy range $6-8 \mathrm{keV}$ and $12-16 \mathrm{keV}$ are presented in Figure 3. It can be observed that the configuration of flaring 

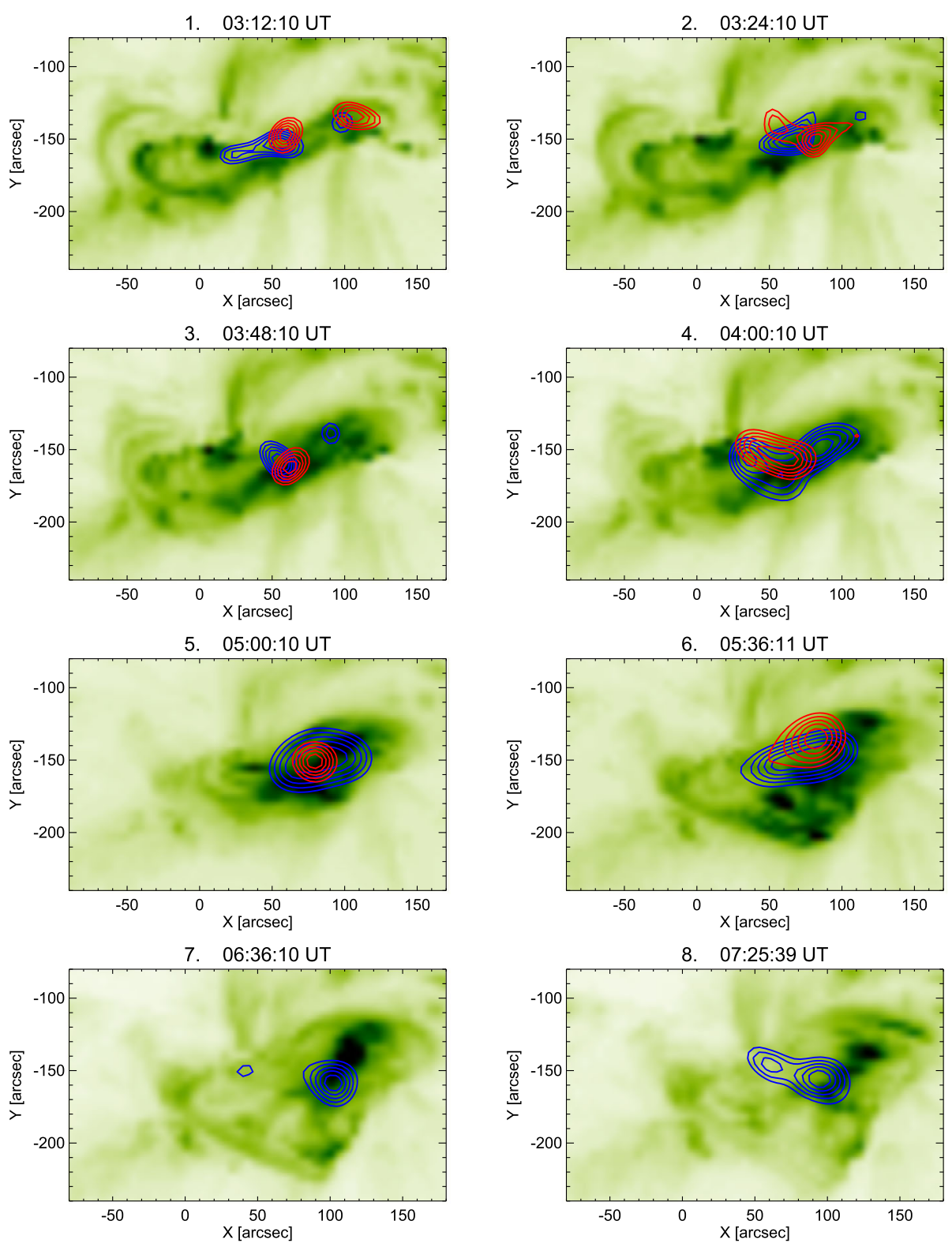

Figure 3 The SOHO/EIT $195 \AA$ A images with RHESSI contours obtained in two energy ranges: 6 - $7 \mathrm{keV}$ (in blue) and $12-16 \mathrm{keV}$ (in red) are presented for selected times. The RHESSI image reconstruction was made using the PIXON method implemented in SolarSoft (grids 3, 4, 5, 6, 8, and 9 have been used).

sources was rather complicated. The emission was still visible a few hours after maximum of the event. At the beginning of the rise phase (image No. 1 in Figure 3) two separate sources are observed, 12 minutes later (image No. 2) only one source is visible. After 04:00 UT, the source becomes much larger (images No. 4, 5, 6). At 05:00 UT (image No. 5) when the second maximum of ultraviolet emission is observed a substantial brightening of the low 
Figure 4 The integrated spectrum of

SOL2002-04-15T03:55 flare (146 minutes of integration time) for the three RESIK channels (1: $3.3-3.8 \AA$, blue;

2: $3.83-4.3 \AA$, red;

4: $5.00 \AA-6.05 \AA$, olive). Due to technical problems at the time of these observations the channel 3 $(4.35-4.86 \AA)$ data are not available for the analysis.

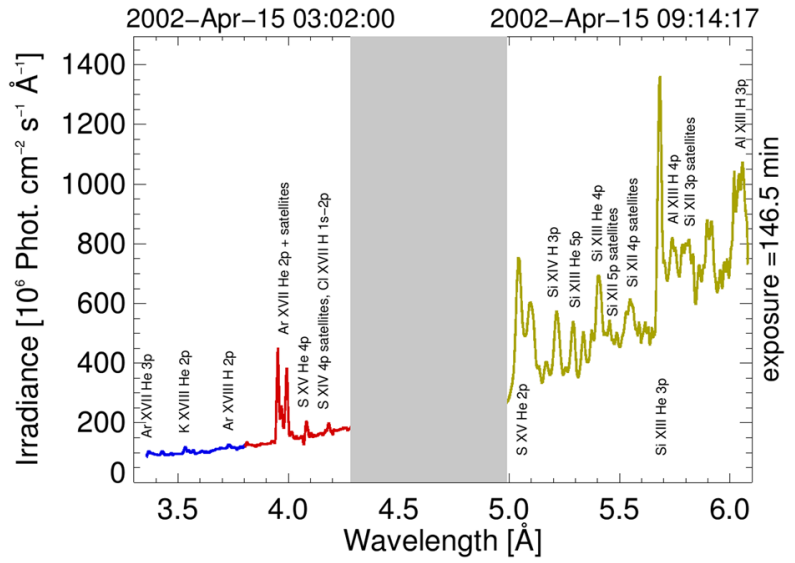

lying loops system on the EIT image can be noticed. The contours of the RHESSI source overlap with the location of the source of ultraviolet emission. The last three images depict the final evolution of this emission system showing a weakening of the observed X-ray source. Starting from 06:30 UT (three hours from X-ray maximum) only the emission at the lower energy range $(6-8 \mathrm{keV})$ can be observed.

RESIK observations are available from 02:45 UT to 09:14 UT with a few breaks when the Coronas- $F$ spacecraft crossed through a polar van Allen radiation belt or the South Atlantic Anomaly when the high voltage of the spectrometer was turned off. However, the first 15 minutes of observation (extending to about 03:00 UT) were associated with the earlier event (see Figure 1).

For the SOL2002-04-15T03:55 flare RESIK obtained almost 900 spectra during about 2.5 hours with cadence of ten seconds. The spectra during the entire rise phase (about 35 minutes) and during more than two hours of decay phase were available. Unfortunately, due to onboard computer set-up problems the channel 3 data (for this flare) were not available. The average RESIK spectrum for SOL2002-04-15T03:55 flare with strong emission lines indicated is presented in Figure 4.

\section{The Analysis}

\subsection{The Isothermal Approximation}

We determined the temporal profiles of temperatures $\left[T_{\mathrm{e}}\right]$ and emission measures $[E M]$ based on GOES X-ray monitor data. These parameters were derived using the isothermal approximation and applying the flux-ratio technique. The pre-flare pedestal was subtracted from the GOES fluxes (0.5-4 $\AA$ and 1-8 $\AA$ channels). The calculations were made using the standard routine goes.pro available in the SolarSoft package. The flaring plasma has reached its maximum temperature, 14.6 MK, at 3:20 UT. About 50 minutes later (at 04:08 UT), the maximum of emission measure equal to $7.6 \times 10^{48} \mathrm{~cm}^{-3}$ was observed. The temporal evolution of temperature and emission measure as calculated based on GOES are presented in Figure 5 (upper-left panel).

An important parameter characterizing the total accumulated energy of flare plasma is the thermal energy [ $\left.E_{\mathrm{th}}\right]$. By definition for the isothermal plasma of density $N_{\mathrm{e}}$ and volume 

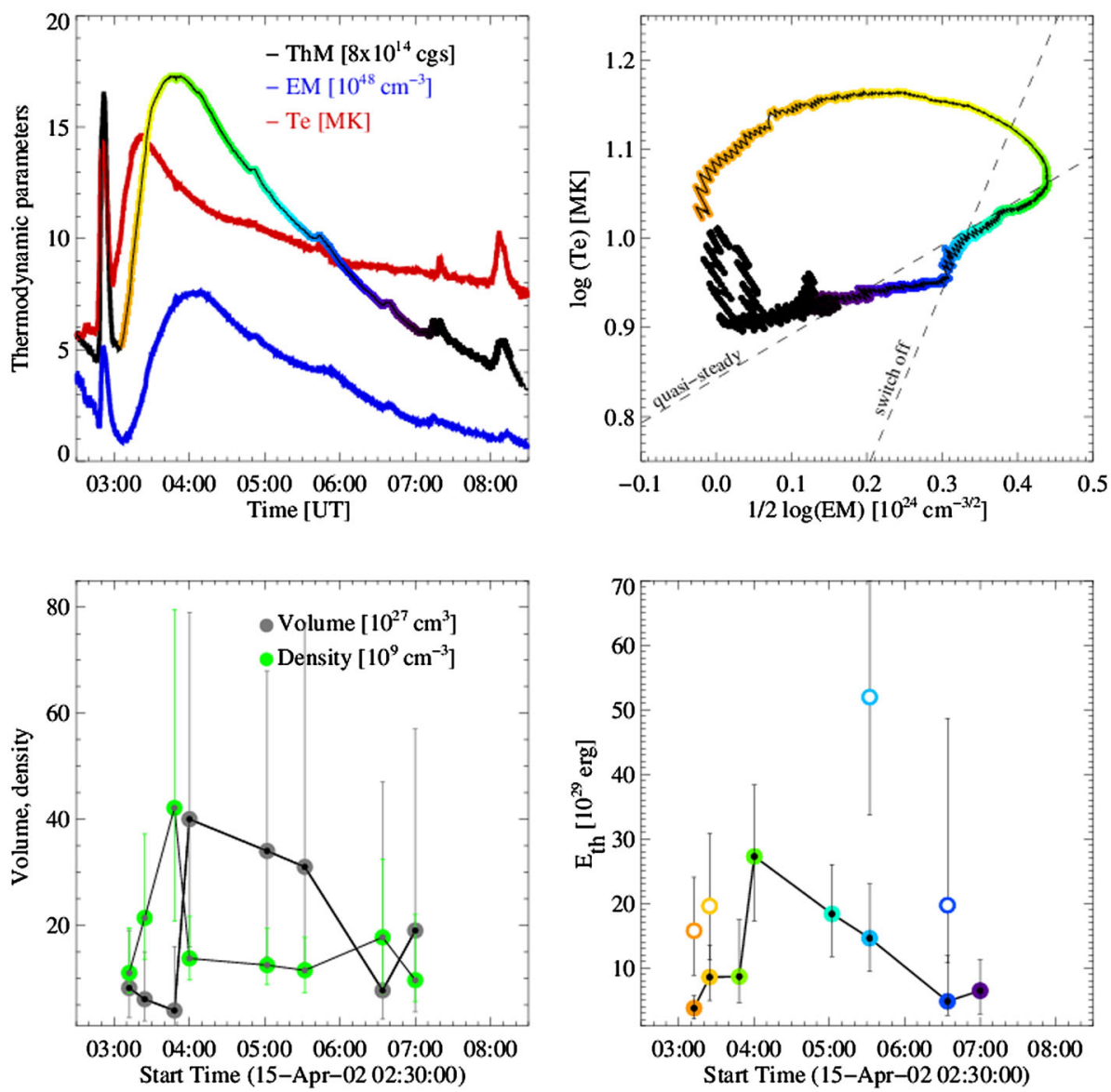

Figure 5 Top-left: The temporal variations of temperature, emission measure, and the thermodynamic measure calculated based on GOES fluxes using the isothermal approximation for the SOL2002-04-15T03:55 flare, right: the diagnostic diagram (DD) for the estimated $T_{\mathrm{e}}$ and $E M$ values. The dashed lines on the DD plot indicate two "limiting cases": quasi-steady state and instant switching off of the heating. Bottom: temporal evolution of flaring volume, average "low density" limit (left) and the total thermal-energy content (right) calculated based on the volume estimated from RHESSI images reconstructed in the $6-8 \mathrm{keV}$ energy range. The colors in the diagnostic diagram correspond to the colors representing the thermodynamic measure (upper-left panel). Circles with a black dot represent thermal energy obtained for isothermal approximation, empty circles represent $E_{\mathrm{th}}$ values calculated based on the differential emission measure distributions and constant pressure assumption (see Equation 6 and the discussion in Sectopm 3.2.3).

$V$ it is:

$$
E_{\text {th }}=3 \mathrm{k} T_{\mathrm{e}} N_{\mathrm{e}} V=3 \mathrm{k} T_{\mathrm{e}} \sqrt{E M} \sqrt{V}[\mathrm{erg}]
$$

where $\mathrm{k}$ is the Boltzmann constant and $E M=N_{\mathrm{e}}^{2} V$.

Provided the flaring plasma volume is constant, we can use so-called thermodynamic measure (ThM), Sylwester, Garcia, and Sylwester (1995).

$$
T h M=3 \mathrm{k} T_{\mathrm{e}} \sqrt{E M}\left[\mathrm{~g} \mathrm{~cm}^{1 / 2} \mathrm{~s}^{-2}\right]
$$


The $T h M$ represents the thermal-energy content in the X-ray plasma source.

$$
E_{\mathrm{th}}=\sqrt{V} T h M[\mathrm{erg}]
$$

Thermal energy can also be determined for multi-temperature plasma assumption. Appropriate equations are presented in the next section. Respective temporal variations of the thermodynamic measure for the analyzed flare are presented on the upper-left panel in Figure 5.

For the eight time intervals, RHESSI images have been reconstructed (see Figure 3), so it was possible to estimate the plasma volume by assuming spherical symmetry of the emitting source, calculate the lower limit of plasma density (filling factor assumed $=1$ ), and determine the total thermal-energy content. The plasma volume was estimated using RHESSI images obtained in the $6-8 \mathrm{keV}$ energy range (the $50 \%$ contour was used in this respect). For the assumed shape of the source, the estimated volumes changed from $3.9 \times$ $10^{27} \mathrm{~cm}^{3}$ to $4 \times 10^{28} \mathrm{~cm}^{3}$. The respective values for the electron density of the hot plasma are $9.6 \times 10^{9} \mathrm{~cm}^{-3}$ and $4.2 \times 10^{10} \mathrm{~cm}^{-3}$.

The amount of thermal energy with the above assumptions is of the order of $10^{30} \mathrm{ergs}$ (see the lower panel of Figure 5). In order to estimate errors in the calculated density and thermal energy, the volumes of the source were determined additionally for contours at $30 \%$ and $70 \%$ of the peak intensity. For the volume of source defined in this way, the obtained values of density and thermal energy differ from those obtained for contour $50 \%$ by a factor of 2 and 1.5 , respectively.

Based on the results of hydrodynamic modeling of the flare (code Palermo-Harvard: PH), Jakimiec et al. (1992) proposed the method for a heating and cooling diagnostic of a flaring loop. This method is based on the analysis of the trajectory slopes on the densitytemperature diagnostic diagram (DD). Two limiting slopes of the flare decay phase were identified that correspond to the situation of a sudden switch off of the flare heating and the evolution along a so-called quasi-steady state line. Intermediate inclinations correspond to the decrease of flaring heating with a decay time $\tau\left[E_{\mathrm{H}}(t) \sim \mathrm{e}^{\frac{-t}{\tau}}\right.$, where $E_{\mathrm{H}}$ is the flare heating rate]. In this article we used a simplified version of the diagnostic diagram in which the density is replaced by the square root of the emission measure (i.e. constant volume of the emitting source is assumed).

Based on the inspection of the diagnostic diagram the timing of the nearly constant heating $[\Delta t]$ has been determined. For the SOL2002-04-15T03:55 flare $\Delta t$ was about one hour. Subsequently the evolution of the flare was along the quasi-steady-state branch.

One must remember that the PH hydrodynamic modeling was performed with the following, rather strong, assumptions:

i) the flaring plasma is confined in a single loop of constant cross section.

ii) the uniform (across the structure) heating is located at the loop top.

In the case of the investigated flare, the morphology is rather complicated, so the results obtained using the diagnostic diagram must be regarded as "indicative".

\subsection{Determination of the Differential Emission Measure Distribution}

\subsubsection{Methods}

The differential emission measure $\left[\varphi\left(T_{\mathrm{e}}\right)\right]$ may loosely be regarded as the amount of the emitting plasma present in a given temperature range (for a physical interpretation of the 
DEM, see, e.g. Sylwester, Mewe, and Schrijver, 1980; Guennou et al., 2012):

$$
\varphi\left(T_{\mathrm{e}}\right)=N_{\mathrm{e}}^{2} \frac{\mathrm{d} V}{\mathrm{~d} T_{\mathrm{e}}}
$$

where: $N_{\mathrm{e}}$ is the electron density, $V$ is the plasma volume, and $T_{\mathrm{e}}$ is the temperature. Its form can be determined by solving a set of integral equations defining the observed line fluxes:

$$
F_{i}=A_{i} \int_{0}^{\infty} f_{i}\left(T_{\mathrm{e}}\right) \varphi\left(T_{\mathrm{e}}\right) \mathrm{d} T_{\mathrm{e}} \quad i=1,2, \ldots, N
$$

where $A_{i}$ represents the assumed abundance of an element contributing to the flux of a particular line or spectral interval, $N$ is the number of spectral bands used, and $f_{i}\left(T_{\mathrm{e}}\right)$ is the theoretically calculated emission function for a given line/band. The emission function represents the flux in the selected line/band at the temperature $T_{\mathrm{e}}$ for a given set of abundances at a distance of $1 \mathrm{AU}$. The emission functions depend on the adopted calculations of the ionization equilibrium and the populations of excited levels associated with the formation of the line.

The idea of determining the DEM is to find such a profile of DEM distribution that provides the most probable agreement between observed and calculated based on this distribution fluxes (the right side of Equation 5).

Both the emission functions and the observed fluxes have uncertainties. It is difficult to estimate the uncertainties of the emission functions. Therefore, it is usually assumed that their values are determined accurately and uncertainties in the resulting calculated distributions of DEM are due to uncertainties in the observed fluxes only.

The reconstruction of differential emission measure distributions is a well-known example of an ill-posed mathematical problem. A direct inversion of the data does not produce a unique DEM solution and additional constraints are needed to achieve a stable solution.

Nowadays, there are a number of methods for inversion of DEM shape from solar and stellar data - see for example Aschwanden et al. (2015). In the present study we show DEM distributions obtained using the two algorithms: Withbroe-Sylwester (WS) and differential evolution (DE).

The Withbroe-Sylwester (maximum likelihood) multiplicative algorithm is an iterative procedure that allows for determination of DEM shape without setting additional conditions on the character of the distribution, and its solution is always positive (Fludra and Sylwester, 1986).

The second method that we used here is based on the mechanisms of differential evolution (DE). Differential evolution (Storn and Price, 1997) is a stochastic evolutionary algorithm used for global optimization. DE is a population-based algorithm like genetic algorithms using similar operators: crossover, mutation, and selection. The distributions of differential emission measure obtained based on genetic algorithm for Hinode data were presented by Siarkowski et al. (2008). The main difference in obtaining a final solution is that genetic algorithms rely on crossover while DE relies on the mutation operation. In the DE method the mutation is based on the differences of randomly sampled pairs of solutions in the population (Storn and Price, 1997). Here, we adopted this approach for the calculation of DEM distributions. DE works with a set of randomly generated individuals (corresponding to (in our case) DEM distributions), representing possible solutions of the problem. 
Table 1 Spectral bands used in calculation of DEMs.

\begin{tabular}{rlll}
\hline No. & Wavelength range $[\AA]]$ & Main line & RESIK channel \\
\hline $\mathbf{1}$ & $3.454-3.466$ & Ar XVI 3p & 1 \\
$\mathbf{2}$ & $3.520-3.542$ & K XVIII $(w)$ & 1 \\
$\mathbf{3}$ & $3.542-3.556$ & K XVIII $(x+y)$ & 1 \\
$\mathbf{4}$ & $3.745-3.759$ & Ar XVIII 2p & 1 \\
$\mathbf{5}$ & $3.941-3.961$ & Ar XVII 2p $(w)$ & 2 \\
$\mathbf{6}$ & $3.961-3.977$ & Ar XVII 2p $(x)$ & 2 \\
$\mathbf{7}$ & $3.977-4.003$ & Ar XVII 2p $(z)$ & 2 \\
$\mathbf{8}$ & $4.093-4.109$ & S XV 4p $(z)$ & 2 \\
$\mathbf{9}$ & $5.009-5.077$ & S XV 2p $(w)$ & 4 \\
$\mathbf{1 0}$ & $5.078-5.120$ & S XV 2p $(x+y)$ & 4 \\
$\mathbf{1 1}$ & $5.198-5.242$ & Si XIV 3p & 4 \\
$\mathbf{1 2}$ & $5.257-5.316$ & Si XIII 5p & 4 \\
$\mathbf{1 3}$ & $5.376-5.426$ & Si XIII 4p & 4 \\
$\mathbf{1 4}$ & $5.526-5.584$ & Si XII sat. & 4 \\
$\mathbf{1 5}$ & $5.659-5.709$ & Si XIII 3p & 4 \\
$\mathbf{1 6}$ & $5.782-5.845$ & Si XII 3p sat & 4 \\
$\mathbf{1 7}$ & $6.046-6.063$ & Al XIII 3p & 4 \\
\hline
\end{tabular}

\subsubsection{Tests of DEM Inversions}

In our previous article (Kepa et al., 2016) we analyzed DEMs for another flare using for the first time the DE approach. In the present work, we reduced the number of temperature intervals and calculate the DEM distributions in the temperature range from 3 to $30 \mathrm{MK}$.

Based on the RESIK spectrum of SOL2002-04-15T03:55 flare (see Figure 4) we have chosen 17 spectral bands covering the respective wavelength intervals. Their main characteristics (wavelength ranges and the most important line contributions) are presented in Table 1. The corresponding emission functions for these spectral bands containing both line and continuum contributions were calculated using the CHIANTI v. 8.1 atomic data package with ionization equilibrium from Bryans, Landi, and Savin (2009). For silicon, sulfur, argon, and potassium we used abundances calculated using the AbuOpt method from multithermal assumption (Sylwester et al., 2015). We assumed $A_{\mathrm{Si}}=4.07 \times 10^{-5}, A_{\mathrm{S}}=2.13 \times 10^{-6}$, $A_{\mathrm{Ar}}=5.49 \times 10^{-6}, A_{\mathrm{K}}=2.95 \times 10^{-7}$. For other elements we adopted abundances from sun_coronal_ext.abund (available in the CHIANTI 8.1 package). Figure 6 (right panel) displays the temporal evolution of RESIK fluxes observed in selected wavelength ranges and respective, theoretically calculated, emission functions (left panel). These data sets were used in determinations of differential emission measure distributions.

To validate the success of reconstruction and assess the quality of DEM calculation we performed a number of tests of the inversion methods used in the present article. In this respect we assumed the shape of a synthetic model of differential emission measure and calculated (from Equation 7) fluxes in the set of basic bands indicated in Table 1. Then each of the calculated fluxes was perturbed by a random error (15\% of the value), and we treated the calculated set as the observed one $\left(F_{\text {obs }}=F_{\text {calc }}+\varepsilon\right.$, where $\varepsilon$ is a random error). Next we used this set of values as input data for the inversion. The results of tests for six selected isoand multi-thermal models of DEM for WS and DE approaches are presented in Figure 7.

The black line represents the assumed synthetic model of DEM and blue lines illustrate 100 results as obtained using the differential evolution method. To do this, each of the flux 

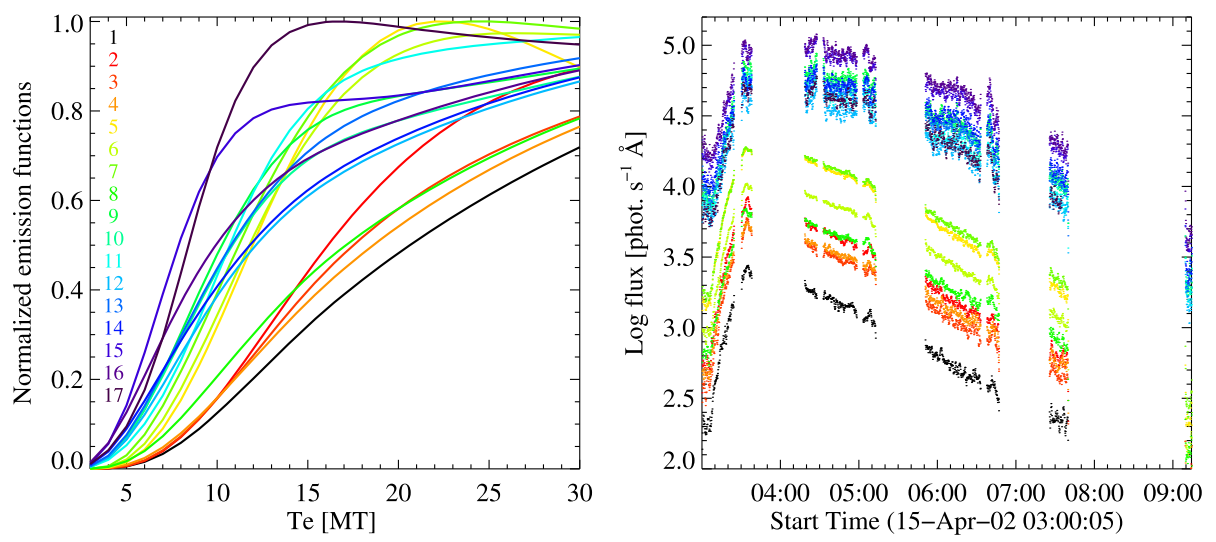

Figure 6 Left: The emission functions for selected 17 wavelength ranges used in the present study. Colored key numbers denoting the curves correspond to bands given in Table 1. Right: The temporal evolution of RESIK fluxes observed at selected wavelength ranges for the investigated flare. These fluxes were used as input data for derivation of differential emission measure distributions.
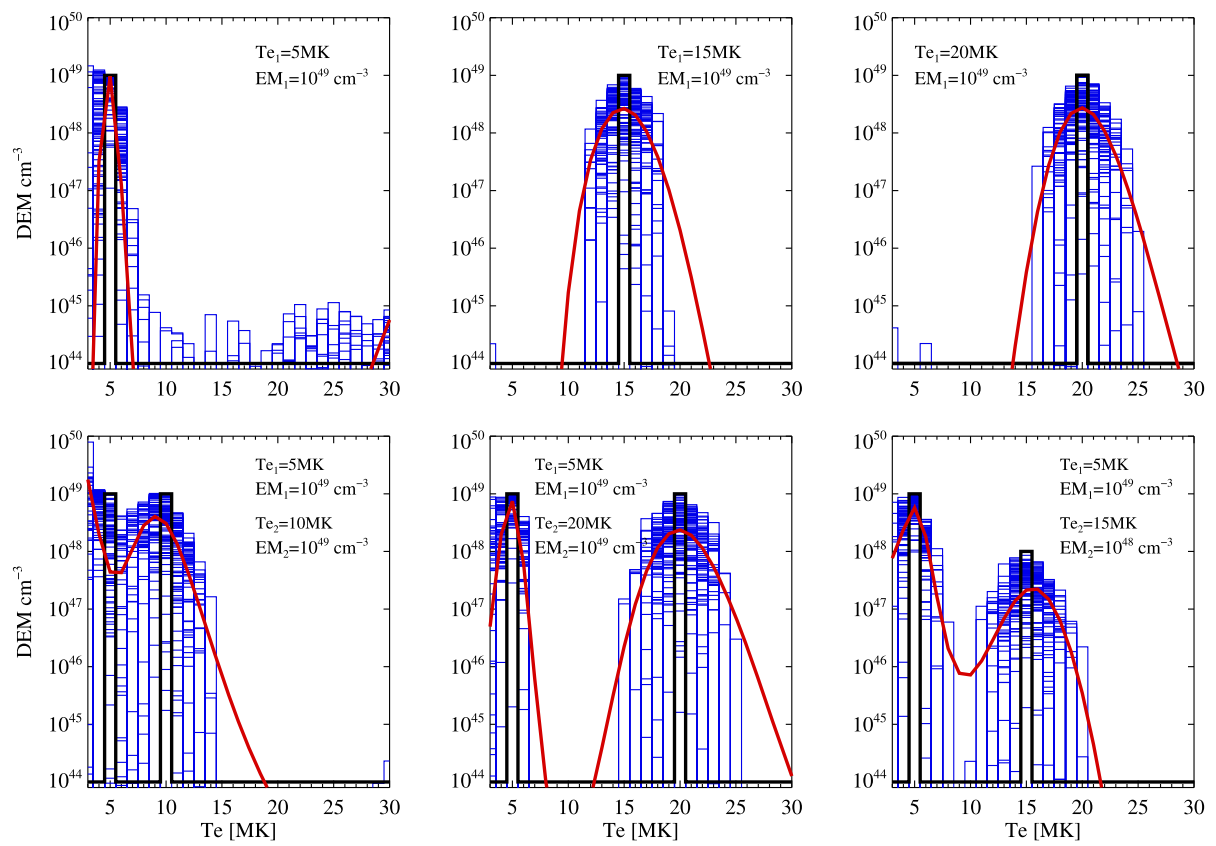

Figure 7 The comparison of assumed (black line) with 100 calculated (blue rectangles) models obtained using the differential evolution (DE) method and Withbroe-Sylwester (WS) approach (in red curves). Upper panel: results for isothermal DEM models at 5, 15 and $20 \mathrm{MK}$; lower panel: for two-temperature synthetic models: the same amount of plasma at $5 \mathrm{MK}$ and $10 \mathrm{MK}, 5$ and $20 \mathrm{MK}$, and with different amount of plasma at 5 and $15 \mathrm{MK}$.

values has been perturbed 100 times, so 100 different sets of the input fluxes and 100 results were obtained. The process of evolution for each restored model was stopped after 10,000 iterations, when the convergence expressed in terms of $\chi^{2}$ became very slow. 

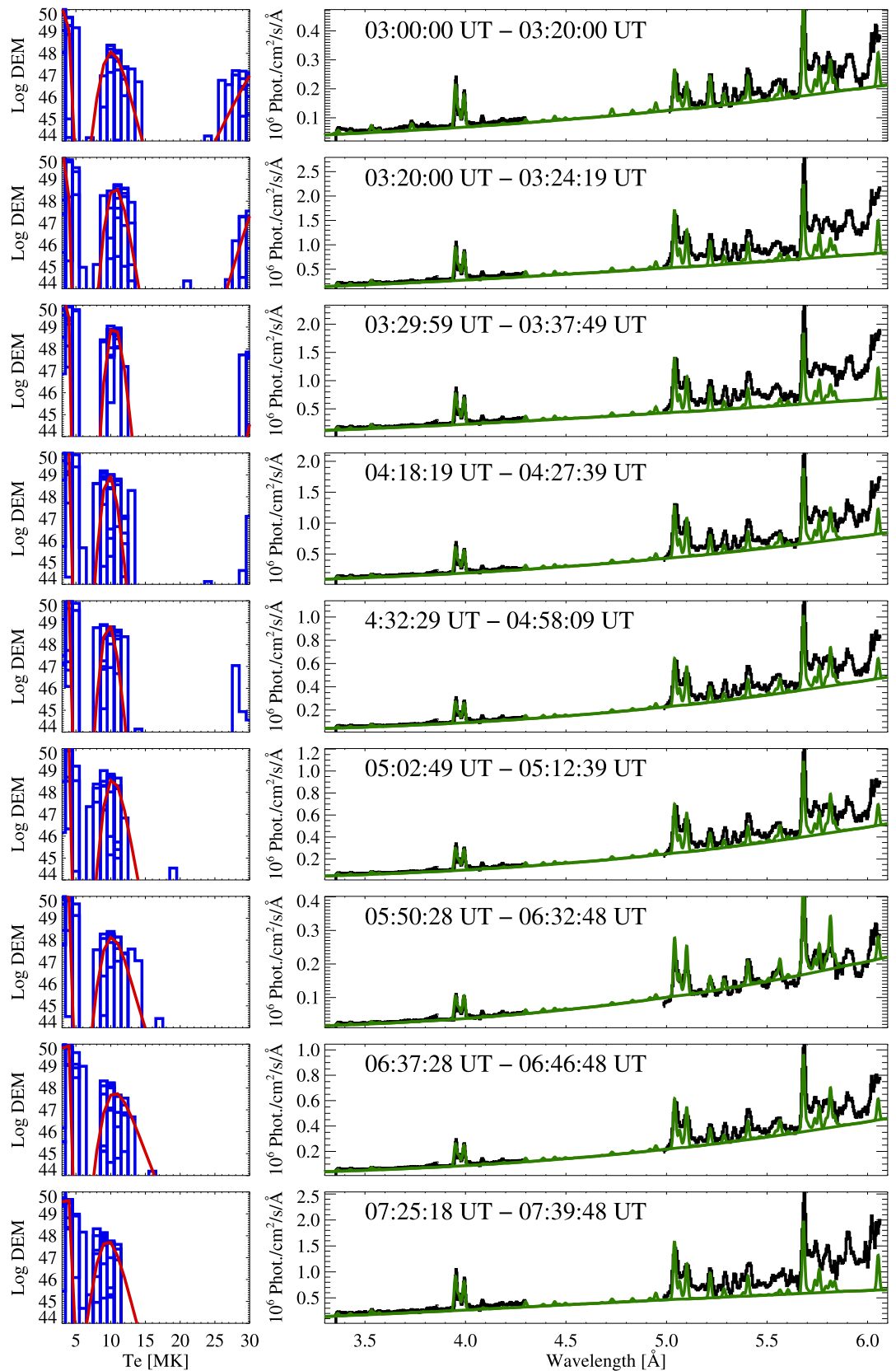

Figure 8 The sequence of derived DEM distributions (left panel) and RESIK measured and calculated spectra (right) taken at nine selected temporal intervals. Each row corresponds to one temporal interval. Colors blue and red are related to difference evolution (DE) and Withbroe-Sylwester (WS) inversion methods, respectively. For each temporal interval for DE ten differential emission measure distributions correspond to ten independent generations of population are presented. In the right panel the RESIK observed spectra are presented in black. Synthetic spectra (in green) were calculated based on best fitted DEM distributions obtained using DE method shown in the left panel. 
For clarity, the results of reconstructions the synthetic models of DEM using the Withbroe-Sylwester method (in red) are presented only for the unperturbed fluxes.

One can see that both DE and WS methods reconstruct satisfactorily the isothermal and two-temperature models.

\subsubsection{DEM Distributions for 15 April 2002 Flare}

Based on RESIK light curves we selected nine temporal intervals for detailed DEM reconstruction. The timings are given in Figure 8 in the right column. For each one we calculated the mean spectrum and fluxes in 17 sets of wavelength ranges (see Table 1). To avoid the contribution of non-flaring plasma, the pre-flare X-ray fluxes have been subtracted. The differential emission measure distributions have been determined using two method described above: Withbroe-Sylwester (WS) and differential evolution (DE). The calculations have been performed in the temperature range 3-30 MK. The results are presented in the left panel of Figure 8. In the right panel we show the comparison of our best fit calculated spectrum with observations. We calculated spectra based on DEM distributions obtained using the DE method and CHIANTI ver. 8.0.1. We used the same ionization balance and set of element abundances as we used in the calculations of emission functions. The discrepancy between observed and synthesized spectra in spectral range 5.9-6 $\AA$ may be associated with the contribution of emissions from higher orders of reflection in RESIK spectra, to the firstorder spectra we observe in the fourth channel. The number of counts at the single detector for each position is equal to the sum of counts from the first order (at emitted wavelength $\lambda$ ), second order (at $\lambda / 2)$, third order $(\lambda / 3)$, etc. For the Si 111 cut mono crystal reflections, used in RESIK for channel 4, the second order of reflection is prohibited, while the allowed third order of reflection includes the He-like Fe line complex (at $1.85 \AA$ ) and the Ni line complex (at $1.55 \AA$ ). The intensity of those lines can influence the emission spectra in channel 4 . Unfortunately those effects are very difficult to account for and the work on this problem is still in progress.

The calculated DEM distributions are two-component independent of the evolutionary phase. Both WS and DE methods give similar results. The small amount of hotter plasma $(25-30 \mathrm{MK})$ is seen at the beginning (rise and maximum) of this long-duration event (Tanaka, 1986).

The temporal evolution of the three components of the differential emission measure distribution is shown in Figure 9. The green, blue, and red plots represent the total emission measure calculated in temperature ranges 3-6 MK, 7-16 MK, and 25-30 MK respectively.

It can be noticed that the temporal behavior of the coldest component (green color) is very similar to the GOES lightcurve shape in the $1-8 \AA$ range. This component is mainly determined by the emission observed in the fourth RESIK channel, where silicon spectral lines are observed. The temporal evolution of hot and hotter components also resemble flare profiles, but they are not correlated with GOES or RHESSI light curves. The hotter component is observed from the rise phase, at maximum, and up to an hour after the maximum of X-ray emission. During the decay phase of the flare, the total amount of plasma associated with this component is almost two orders smaller than just before the maximum.

Based on the calculated differential emission measure distributions and the assumptions of constant pressure (see Equation 6) or constant plasma density (see Equation 7) the thermal-energy content can be calculated.

$$
E_{\mathrm{th} \mid p=\text { const }}=3 \mathrm{k} \sqrt{V} \sqrt{\int T^{2} \varphi\left(T_{\mathrm{e}}\right) \mathrm{d} T_{\mathrm{e}}}
$$




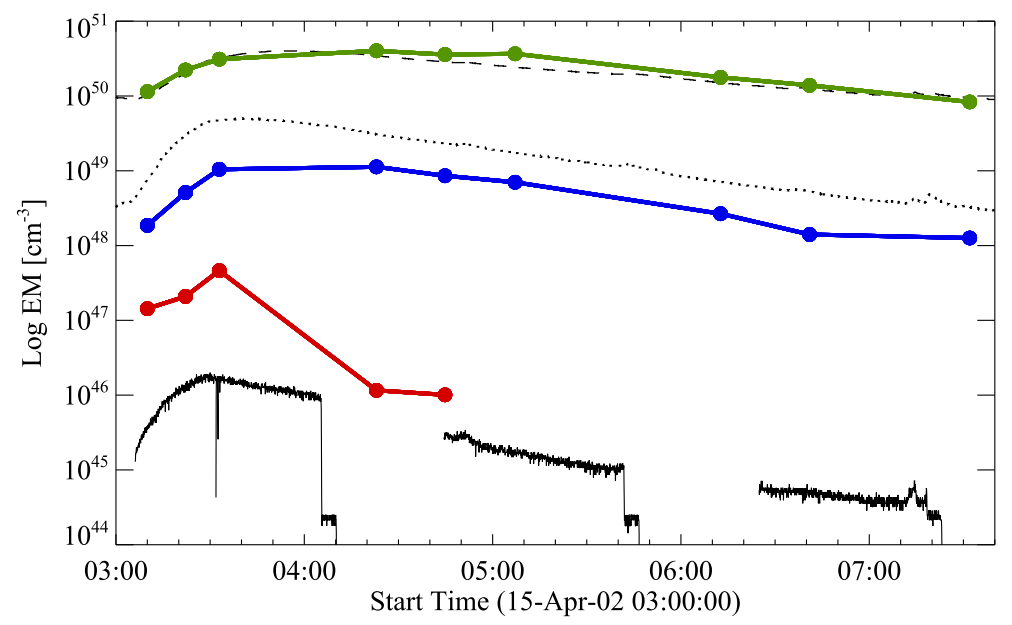

Figure 9 The temporal evolution of the three components of the differential emission measure as calculated based on average DEM distributions using DE and WS methods. The green, blue, and red represent total emission measure present in the temperature ranges 3-6 MK, 7-16 MK, and 25-30 MK respectively. The thin dashed and dotted lines present the GOES light curves in the $1-8 \AA$ and $0.5-4 \AA$ ranges. The bottom black line is the RHESSI lightcurve in the 6-12 keV energy range. The GOES and RHESSI light curves have been scaled for better visualization.

$$
E_{\mathrm{th} \mid N_{\mathrm{e}}=\text { const }}=3 \mathrm{k} \sqrt{V} \frac{\int T_{\mathrm{e}} \varphi\left(T_{\mathrm{e}}\right) \mathrm{d} T_{\mathrm{e}}}{\sqrt{\int \varphi\left(T_{\mathrm{e}}\right) \mathrm{d} T_{\mathrm{e}}}}
$$

We determined the thermal-energy content for four temporal intervals for which RHESSI images and RESIK observations were available. Our calculations have been performed for both constant pressure and constant density assumptions. The results obtained are similar, so $E_{\mathrm{th}}$ values calculated for the constant pressure assumption have been used only. Obtained results are presented in Figure 5 (bottom-right panel). The values are higher than for an isothermal model, but the results agree within an order of magnitude $\left(10^{30} \mathrm{ergs}\right)$.

\section{Concluding Remarks}

Based on the data from GOES, RHESSI, SOHO/EIT, and RESIK we carried out analysis of the long-duration event that occurred on 15 April 2002 (SOL2002-04-15T03:55). We determined physical characteristics of flaring plasma (temperature, emission measure, thermodynamic measure, density, thermal-energy, and differential emission measure distributions) and investigated their temporal evolutions based on GOES, RHESSI, SOHO/EIT, and RESIK data.

The obtained results are consistent with the knowledge about the long-duration flares. A novelty in the work is the application of the differential evolution method to determine the differential emission measure distributions. For the first time, this approach was tested on synthetic models and compared with the Withbroe-Sylwester method.

Our analysis can be summarized as follows: 
i) The ranges of flaring plasma temperature and emission measure calculated in an isothermal approximation based on GOES fluxes are 7.9-14.6 MK and $1 \times 10^{48}-7.6 \times$ $10^{48} \mathrm{~cm}^{-3}$ respectively.

ii) The size of the source is variable during the flare evolution. From RHESSI images reconstructed in the energy range $6-8 \mathrm{keV}$ and assuming the equivalent spherical shape of the X-ray source, the calculated volume changes from: $3.9 \times 10^{27} \mathrm{~cm}^{3}$ to $4.0 \times 10^{29} \mathrm{~cm}^{3}$.

iii) The corresponding range of densities for the hot plasma component is $9.6 \times 10^{9}-4.3 \times$ $10^{10} \mathrm{~cm}^{-3}$.

iv) The differential emission measure distributions were calculated based on two methods: Withbroe-Sylwester and differential evolution. Both of the methods were tested. The results of the tests confirmed the stability of the solutions and capability to reconstruct the synthetic distributions in the specified temperature range from 3 to $30 \mathrm{MK}$. Both WS and DE methods provide similar DEM inversions.

v) The obtained DEM distributions are always two-component independent of the evolutionary phase. A small amount (the third component) of hotter plasma $(25-30 \mathrm{MK})$ is seen at the beginning (rise and maximum) of this long-duration event. This component may be associated with the presence of suprathermal electrons based on the coincidence with the harder X-rays.

vi) The temporal evolution of the coldest component (green color in Figure 9) mimics the evolution of the GOES flux in the $1-8 \AA$ range.

vii) The amount of thermal plasma energy content is of the order of $10^{30} \mathrm{ergs}$. The values calculated by the assuming isothermal plasma model are lower than those calculated based on the differential emission measure distributions.

Acknowledgements We acknowledge financial support from the Polish National Science Centre grants No. 2017/25/B/ST9/01821 and 2015/19/ST9/02826.)

Disclosure of Potential Conflicts of Interest The authors declare that they have no conflicts of interest.

Publisher's Note Springer Nature remains neutral with regard to jurisdictional claims in published maps and institutional affiliations.

Open Access This article is licensed under a Creative Commons Attribution 4.0 International License, which permits use, sharing, adaptation, distribution and reproduction in any medium or format, as long as you give appropriate credit to the original author(s) and the source, provide a link to the Creative Commons licence, and indicate if changes were made. The images or other third party material in this article are included in the article's Creative Commons licence, unless indicated otherwise in a credit line to the material. If material is not included in the article's Creative Commons licence and your intended use is not permitted by statutory regulation or exceeds the permitted use, you will need to obtain permission directly from the copyright holder. To view a copy of this licence, visit http://creativecommons.org/licenses/by/4.0/.

\section{References}

Aschwanden, M.J., Wuelser, J.P., Nitta, N.V., Lemen, J.R.: 2009, Solar flare and CME observations with STEREO/EUVI. Solar Phys. 256, 3. DOI. ADS.

Aschwanden, M.J., Boerner, P., Caspi, A., McTiernan, J.M., Ryan, D., Warren, H.: 2015, Benchmark test of differential emission measure codes and multi-thermal energies in solar active regions. Solar Phys. 290, 2733. DOI. ADS.

Ba̧k-Stȩślicka, U., Mrozek, T., Kołomański, S.: 2011, Energy release during slow long-duration flares observed by RHESSI. Solar Phys. 271, 75. DOI. ADS. 
Bryans, P., Landi, E., Savin, D.W.: 2009, A new approach to analyzing solar coronal spectra and updated collisional ionization equilibrium calculations. II. Updated ionization rate coefficients. Astrophys. J. 691, 1540. DOI. ADS.

Chifor, C., Del Zanna, G., Mason, H.E., Sylwester, J., Sylwester, B., Phillips, K.J.H.: 2007, A benchmark study for CHIANTI based on RESIK solar flare spectra. Astron. Astrophys. 462, 323. DOI. ADS.

Delaboudinière, J.-P., Artzner, G.E., Brunaud, J., Gabriel, A.H., Hochedez, J.F., Millier, F., Song, X.Y., Au, B., Dere, K.P., Howard, R.A., Kreplin, R., Michels, D.J., Moses, J.D., Defise, J.M., Jamar, C., Rochus, P., Chauvineau, J.P., Marioge, J.P., Catura, R.C., Lemen, J.R., Shing, L., Stern, R.A., Gurman, J.B., Neupert, W.M., Maucherat, A., Clette, F., Cugnon, P., van Dessel, E.L.: 1995, EIT: Extreme-Ultraviolet Imaging Telescope for the SOHO mission. Solar Phys. 162, 291. DOI. ADS.

Fludra, A., Sylwester, J.: 1986, Comparison of three methods used for calculation of the differential emission measure. Solar Phys. 105, 323. DOI. ADS.

Guennou, C., Auchère, F., Soubrié, E., Bocchialini, K., Parenti, S., Barbey, N.: 2012, On the accuracy of the differential emission measure diagnostics of solar plasmas. Application to SDO/AIA. II. Multithermal plasmas. Astrophys. J. Suppl. 203, 26. DOI. ADS.

Howard, R.A., Moses, J.D., Vourlidas, A., Newmark, J.S., Socker, D.G., Plunkett, S.P., Korendyke, C.M., Cook, J.W., Hurley, A., Davila, J.M., Thompson, W.T., St Cyr, O.C., Mentzell, E., Mehalick, K., Lemen, J.R., Wuelser, J.P., Duncan, D.W., Tarbell, T.D., Wolfson, C.J., Moore, A., Harrison, R.A., Waltham, N.R., Lang, J., Davis, C.J., Eyles, C.J., Mapson-Menard, H., Simnett, G.M., Halain, J.P., Defise, J.M., Mazy, E., Rochus, P., Mercier, R., Ravet, M.F., Delmotte, F., Auchere, F., Delaboudiniere, J.P., Bothmer, V., Deutsch, W., Wang, D., Rich, N., Cooper, S., Stephens, V., Maahs, G., Baugh, R., McMullin, D., Carter, T.: 2008, Sun Earth connection coronal and heliospheric investigation (SECCHI). Space Sci. Rev. 136, 67. DOI. ADS.

Hurford, G.J., Schmahl, E.J., Schwartz, R.A., Conway, A.J., Aschwanden, M.J., Csillaghy, A., Dennis, B.R., Johns-Krull, C., Krucker, S., Lin, R.P., McTiernan, J., Metcalf, T.R., Sato, J., Smith, D.M.: 2002, The RHESSI imaging concept. Solar Phys. 210, 61. DOI. ADS.

Jakimiec, J., Sylwester, B., Sylwester, J., Serio, S., Peres, G., Reale, F.: 1992, Dynamics of flaring loops. II Flare evolution in the density-temperature diagram. Astron. Astrophys. 253, 269. ADS.

Jakimiec, J., Tomczak, M., Fludra, A., Falewicz, R.: 1997, Energy release and transport in arcade flares. Adv. Space Res. 20, 2341. DOI. ADS.

Jiang, Y.W., Liu, S., Liu, W., Petrosian, V.: 2006, Evolution of the loop-top source of solar flares: heating and cooling processes. Astrophys. J. 638, 1140. DOI. ADS.

Kahler, S.: 1977, The morphological and statistical properties of solar X-ray events with long decay times. Astrophys. J. 214, 891. DOI. ADS.

Kaiser, M.L., Kucera, T.A., Davila, J.M., St. Cyr, O.C., Guhathakurta, M., Christian, E.: 2008, The STEREO mission: an introduction. Space Sci. Rev. 136, 5. DOI. ADS.

Kepa, A., Sylwester, J., Sylwester, B., Siarkowski, M., Stepanov, A.I.: 2006, Determination of differential emission measure from X-ray solar spectra registered by RESIK aboard CORONAS-F. Solar Syst. Res. 40, 294. DOI. ADS.

Kepa, A., Sylwester, B., Sylwester, J., Siarkowski, M., Mrozek, T., Gryciuk, M.: 2016, Multitemperature analysis of solar flare observed on 2003 March 29. In: Kosovichev, A.G., Hawley, S.L., Heinzel, P. (eds.) Solar and Stellar Flares and Their Effects on Planets, IAU Sym. 320, 86. DOI. ADS.

Kolomański, S., Jakimiec, J.: 2002, Analysis of the long-duration arcade flare of 27 April 1998. In: Wilson, A. (ed.) Solar Variability: From Core to Outer Frontiers SP-506, ESA, Noordwijk, 665. ADS.

Kuznetsov, V.D.: 2014, CORONAS-F project: the study of solar activity and its effects on the Earth. In: Kuznetsov, V. (ed.) The Coronas-F Space Mission, Astrophys. Space Sci. Lib. 400, 1. DOI. ADS.

Lin, R.P., Dennis, B.R., Hurford, G.J., Smith, D.M., Zehnder, A., Harvey, P.R., Curtis, D.W., Pankow, D., Turin, P., Bester, M., Csillaghy, A., Lewis, M., Madden, N., van Beek, H.F., Appleby, M., Raudorf, T., McTiernan, J., Ramaty, R., Schmahl, E., Schwartz, R., Krucker, S., Abiad, R., Quinn, T., Berg, P., Hashii, M., Sterling, R., Jackson, R., Pratt, R., Campbell, R.D., Malone, D., Landis, D., BarringtonLeigh, C.P., Slassi-Sennou, S., Cork, C., Clark, D., Amato, D., Orwig, L., Boyle, R., Banks, I.S., Shirey, K., Tolbert, A.K., Zarro, D., Snow, F., Thomsen, K., Henneck, R., McHedlishvili, A., Ming, P., Fivian, M., Jordan, J., Wanner, R., Crubb, J., Preble, J., Matranga, M., Benz, A., Hudson, H., Canfield, R.C., Holman, G.D., Crannell, C., Kosugi, T., Emslie, A.G., Vilmer, N., Brown, J.C., Johns-Krull, C., Aschwanden, M., Metcalf, T., Conway, A.: 2002, The Reuven Ramaty High-Energy Solar Spectroscopic Imager (RHESSI). Solar Phys. 210, 3. DOI. ADS.

Siarkowski, M., Falewicz, R., Kepa, A., Rudawy, P.: 2008, Diagnostic of the temperature and differential emission measure (DEM) based on Hinode/XRT data. Ann. Geophys. 26, 2999. DOI. ADS.

Storn, R., Price, K.: 1997, Differential evolution-a simple and efficient heuristic for global optimization over continuous spaces. J. Glob. Optim. 11, 341. DOI.

Svestka, Z.: 1983, Post-flare coronal arches. Space Sci. Rev. 35, 259. DOI. ADS. 
Sylwester, J., Garcia, H.A., Sylwester, B.: 1995, Quantitative interpretation of GOES soft X-ray measurements. I. The isothermal approximation: application of various atomic data. Astron. Astrophys. 293, 577. ADS.

Sylwester, J., Mewe, R., Schrijver, J.: 1980, Analysis of X-ray line spectra from a transient plasma under solar flare conditions - part three - diagnostics for measuring electron temperature and density. Astron. Astrophys. Suppl. 40, 335. ADS.

Sylwester, B., Sylwester, J., Phillips, K.J.H.: 2008, X-ray studies of flaring plasma. J. Astrophys. Astron. 29, 147. DOI. ADS.

Sylwester, J., Gaicki, I., Kordylewski, Z., Kowaliński, M., Nowak, S., Płocieniak, S., Siarkowski, M., Sylwester, B., Trzebiński, W., Bakała, J., Culhane, J.L., Whyndham, M., Bentley, R.D., Guttridge, P.R., Phillips, K.J.H., Lang, J., Brown, C.M., Doschek, G.A., Kuznetsov, V.D., Oraevsky, V.N., Stepanov, A.I., Lisin, D.V.: 2005, Resik: a bent crystal X-ray spectrometer for studies of solar coronal plasma composition. Solar Phys. 226, 45. DOI. ADS.

Sylwester, B., Phillips, K.J.H., Sylwester, J., Kȩpa, A.: 2015, Resik solar X-ray flare element abundances on a non-isothermal assumption. Astrophys. J. 805, 49. DOI. ADS.

Tanaka, K.: 1986, High-energy observations of solar flares. Astrophys. Space Sci. 118, 101. DOI. ADS.

Woods, T.N., Hock, R., Eparvier, F., Jones, A.R., Chamberlin, P.C., Klimchuk, J.A., Didkovsky, L., Judge, D., Mariska, J., Warren, H., Schrijver, C.J., Webb, D.F., Bailey, S., Tobiska, W.K.: 2011, New solar extreme-ultraviolet irradiance observations during flares. Astrophys. J. 739, 59. DOI. ADS. 